\title{
Percutaneous coronary intervention versus optimal medical therapy for patients with chronic total occlusion: a meta-analysis and systematic review
}

\author{
Yingxu Ma ${ }^{1 \#}$, Dongping $\mathrm{Li}^{2 \#}$, Jiayi $\mathrm{Li}^{1}$, Yixi $\mathrm{Li}^{1}$, Fan Bai ${ }^{1}$, Fen Qin ${ }^{1}$, Shenghua Zhou ${ }^{1}$, Qiming Liu ${ }^{1}$ \\ ${ }^{1}$ Department of Cardiology, ${ }^{2}$ Department of Cardiothoracic Surgery, The Second Xiangya Hospital, Central South University, Changsha 410011, \\ China \\ Contributions: (I) Conception and design: Q Liu; (II) Administrative support: S Zhou; (III) Provision of study materials or patients: Y Ma, D Li; \\ (IV) Collection and assembly of data: Y Ma, J Li; (V) Data analysis and interpretation: D Li, Y Li; (VI) Manuscript writing: All authors; (VII) Final \\ approval of manuscript: All authors. \\ \# These authors contributed equally to this work. \\ Correspondence to: Qiming Liu, MD, PhD. Department of Cardiology, The Second Xiangya Hospital, Central South University, No. 139 Middle \\ Renmin Road, Changsha 410011, China. Email: qimingliu@csu.edu.cn.
}

\begin{abstract}
Background: It was under debate whether chronic total occlusion (CTO) patients could benefit from percutaneous coronary intervention (PCI). We sought to compare clinical outcomes of PCI and optimal medical therapy (OMT) in these patients.

Methods: PubMed, Embase and Cochrane Library were searched for studies enrolling patients with CTO who accepted PCI or OMT. The meta-analysis was performed by using a random-effect model. In addition, subgroup analyses were performed, including patients after propensity-matching and patients with CTO in infarct-related artery (IRA), respectively.

Results: We identified 5 studies enrolling 4,761 participants in this meta-analysis. In the main analysis, when compared with OMT, PCI was associated with significant improvement in all-cause death [risk ratio (RR) 0.41, 95\% CI: 0.35-0.48], cardiac death (RR 0.44, 95\% CI: 0.35-0.55) and major adverse cardiac events (MACE) (RR 0.64, 95\% CI: 0.43-0.97). But there were no differences in myocardial infarction (MI) and stroke. The results of the propensity-matched subgroup were somewhat consistent with those of the main analysis (all-cause death: RR 0.57, 95\% CI: 0.26-0.89; MI: RR 0.54, 95\% CI: 0.32-0.77; and MACE: RR 0.76, 95\% CI: 0.33-1.18). In IRA subgroup, PCI reduced risks of mortality (all-cause death: RR 0.41, 95\% CI: 0.34-0.49; cardiac death: RR 0.44, 95\% CI: 0.35-0.56) and MACE (RR 0.71, 95\% CI: 0.46-1.10). But no difference was observed in MI.
\end{abstract}

Conclusions: PCI was associated with improved survival and reduced MACE relative to OMT.

Keywords: Chronic total occlusion (CTO); percutaneous coronary intervention (PCI); optimal medical therapy (OMT)

Submitted Jan 12, 2018. Accepted for publication Apr 16, 2018.

doi: $10.21037 /$ jtd.2018.04.140

View this article at: http://dx.doi.org/10.21037/jtd.2018.04.140

\section{Introduction}

Chronic total occlusion (CTO) is defined as an obstruction of a coronary artery with Thrombolysis in Myocardial Infarction (TIMI) flow grade 0 for a duration $>3$ months $(1,2)$. The real prevalence of CTO lesions is difficult to be confirmed because a large proportion of patients with CTO lack symptoms and are not referred to physician evaluation. In some registry studies $(3,4)$, the prevalence of CTO among patients undergoing coronary artery angiography was about $18.4-52 \%$. Unfortunately, the fact that most patients were devoid of obvious symptoms and signs (3) contributed to delayed diagnosis and treatment of CTO. What's worse 
was that 1-year mortality in patients with CTO was higher than that in patients without CTO and prognosis was even worse when more than one CTO lesion was found (5). Due to relatively high prevalence and poor prognosis, physicians greatly concerned CTO treatment strategies.

Although guidelines $(1,2)$ recommended that percutaneous coronary intervention (PCI) should be considered in patients with CTO in order to relieve angina and improve life quality, it was under debate whether PCI could benefit CTO patients when compared with optimal medical therapy (OMT). Firstly, CTO procedures which sought for specific equipment and experienced operators were complex, resulting in quite different success rates of CTO procedures across different centers $(6,7)$. Secondly, long CTO procedure time and the need for large amounts of contrast were related to elevated radiation exposure and increased risk of procedural complications. Thirdly, the results of clinical studies involving CTO patients were controversial. The EXPLORE trial (8), the only one published randomized controlled trial to evaluate the impact of PCI on patients with CTO, found negative results and demonstrated that PCI could not improve left ventricular ejection fraction (LVEF) and reduce the risk of major adverse cardiac events (MACE) when compared with OMT. What's more surprising was that the proportion of patients who accepted repeat PCI in PCI group was higher than that in OMT group (26.4\% vs. $13.0 \%, \mathrm{P}=0.004)$ after 4-month follow-up. However, some cohort studies $(6,9,10)$ indicated that PCI was associated with improved long-term survival and better prognosis relative to OMT. Therefore, cardiologists needed more data to help them determine the initial management strategies of CTO. In this metaanalysis, we aimed to compare the outcomes of PCI and OMT in CTO patients that were diagnosed by coronary angiography to offer physicians a more comprehensive picture of therapy strategies in patients with CTO.

\section{Methods}

\section{Search strategy and study selection criteria}

Two investigators independently searched PubMed, Embase and Cochrane Library for studies enrolling CTO patients undergoing PCI or OMT as an initial management strategy, which were published before July 31st 2017 and restricted to English. Our search strategy in PubMed incorporated the Cochrane Highly Sensitive Search Strategy (11). The main search terms were "CTO", "PCI" and "medical therapy" (details of search strategy included in supplement materials). Two authors respectively performed the screening of titles, abstracts or full texts and determined their eligibility. We also searched the reference lists of the original articles identified for full text review to find other eligible studies.

\section{Data extraction}

Two independent reviewers extracted data from included studies. Data extracted from studies included study characteristics, patient characteristics, details regarding PCI and OMT groups, and outcome measures. Outcomes were all-cause death, cardiac death, myocardial infarction (MI), stroke, and MACE, defined as a composite of cardiac death, MI, stroke, heart failure, and revascularization.

\section{Statistical analysis}

Intention-to-treat meta-analysis was performed by Stata 12.0. The main analysis included all studies eligible for this meta-analysis. Propensity-matched analysis could balance the baseline characteristics between groups, increasing the comparability of two groups and reducing bias (12). Four studies (13-16) adopted this approach to perform a secondary analysis to achieve the effect of randomization. Thus, these four studies were included in the propensitymatched subgroup. COREA-AMI study (15) enrolled patients with CTO in non-infarct-related artery (IRA) while another four studies $(13,14,16,17)$ included patients with CTO in IRA. In order to minimize the impacts of different CTO locations on interpretation of the results, these four studies $(13,14,16,17)$ were included in the IRA subgroup.

Outcome data were extracted as risk ratios (RRs) and 95\% confidence intervals (CIs) for PCI versus OMT among patients with CTO. We reported unadjusted RRs since RRs were obtained from different models in two studies $(14,15)$ and three studies $(13,16,17)$ didn't report the adjusted RRs. RRs were pooled in a random-effect model to compute summary effect sizes of outcome data of PCI versus OMT in patients with CTO. Each study estimate of the relative treatment was given a weight that was equal to the inverse of the variance of the effect estimate. The Cochran's Q test and $\mathrm{I}^{2}$ test were performed to assess the heterogeneity of the summary effects. If the $\mathrm{P}$ value of Cochran's $\mathrm{Q}$ test was $<0.10$ and $\mathrm{I}^{2}$ was $>50 \%$, heterogeneity was considered to exist (18). Notably, we excluded two studies $(13,16)$ when we calculated pooled RR of stroke in the main analysis because stroke cases were not reported. In propensity-matched subgroup, 


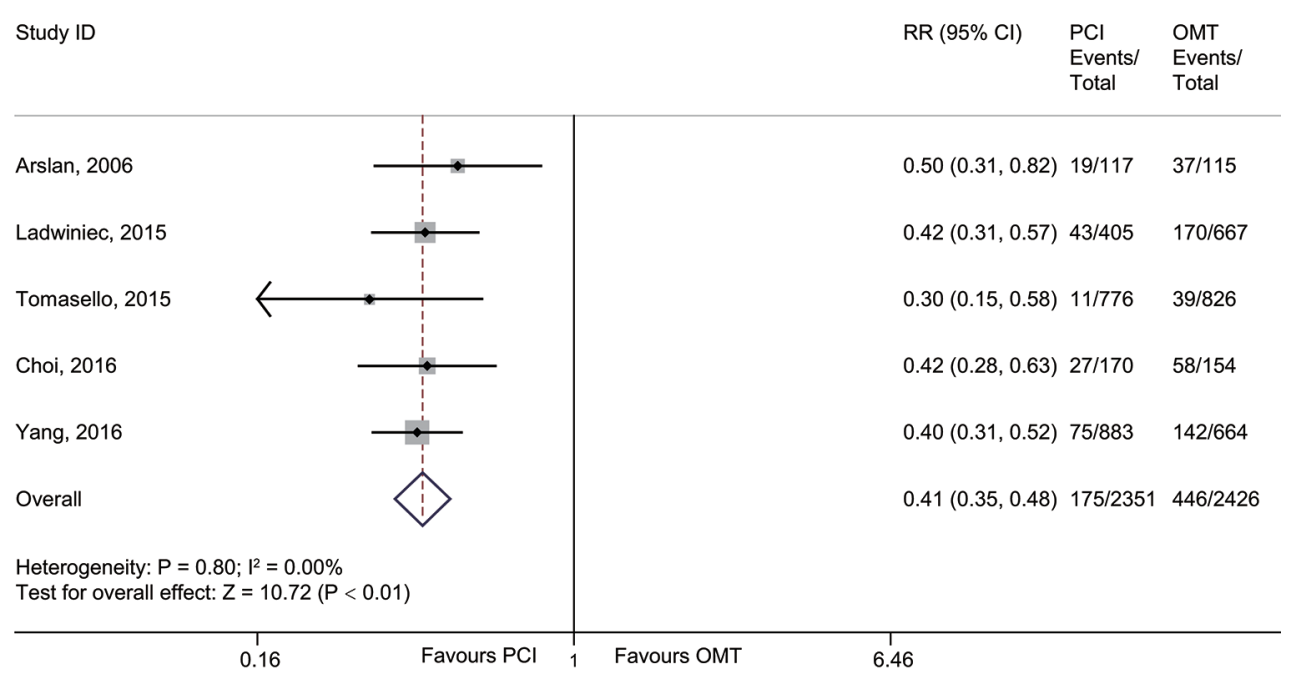

Figure 1 Forest plot with individual and summary estimates of the RR and 95\% CI of all-cause death in the main analysis. RR, risk ratio; CI, confidence interval; PCI, percutaneous coronary intervention; OMT, optimal medical therapy.

we compared only three outcomes between two groups, which incorporated cardiac death, MI and MACE. This was because Tomasello et al. (14) didn't report all-cause death and stroke cases in propensity-matched analysis while another two studies $(13,16)$ didn't report stroke cases. Moreover, we didn't calculate pooled RR of stroke in IRA subgroup because only two studies $(14,17)$ reported the stroke cases.

Publication bias were assessed with funnel plot, Begg's test and Egger's test, respectively. Whether effect sizes were distributed symmetrically or not was judged visually. If $\mathrm{P}$ values of Begg's test and Egger's test were $<0.10$, publication bias was considered to exist.

\section{Sensitivity analyses and quality assessment}

Jackknife sensitivity analyses were performed for each outcome of interest to verify the robustness of the results and the impact of each single study on the summary estimate of the effect. Pooled estimates were recalculated multiple times by using a random-effect model, each time with removal of a single study from the baseline group. Quality of the included studies was assessed by the Newcastle-Ottawa scale (NOS) (19).

\section{Results}

\section{Study characteristics}

The results of study selection process were shown in
Figure S1. One thousand three hundred and fortytwo studies were found in PubMed and three thousand seven hundred and forty studies were found in Embase. Meanwhile, one hundred and fifty records were gotten from Cochrane Library. After exclusion of duplicates, irrelevant studies and studies without corresponding data, we eventually identified five studies (13-17) that fulfilled the inclusion criteria.

The baseline characteristics of populations included in this meta-analysis were shown in Table S1. Of 4,761 patients enrolled in this analysis, CTO was treated by PCI in 2,351 and OMT in 2,410. Patients managed with OMT were older in comparison with patients treated with PCI $(\mathrm{P}<0.05)$ in four studies $(13-16)$. The proportions of males in the included studies were high, ranging from $66.2 \%$ to $84.8 \%$. And there were no significant differences in diabetes, hypertension and history of smoking between PCI and OMT groups in the included studies. The proportions of patients with CTO in left main coronary artery and its branches in PCI groups were higher than those in OMT groups while proportions of patients with CTO in right coronary artery in PCI groups were a bit lower.

\section{Clinical outcomes and subgroup analyses}

In the main analysis, PCI significantly reduced risks of allcause death (RR $0.41,95 \%$ CI: $0.35-0.48$ ) and cardiac death (RR $0.44,95 \%$ CI: $0.35-0.55$ ) by about $60 \%$ as compared with OMT (Figures 1,2). However, there was no difference 


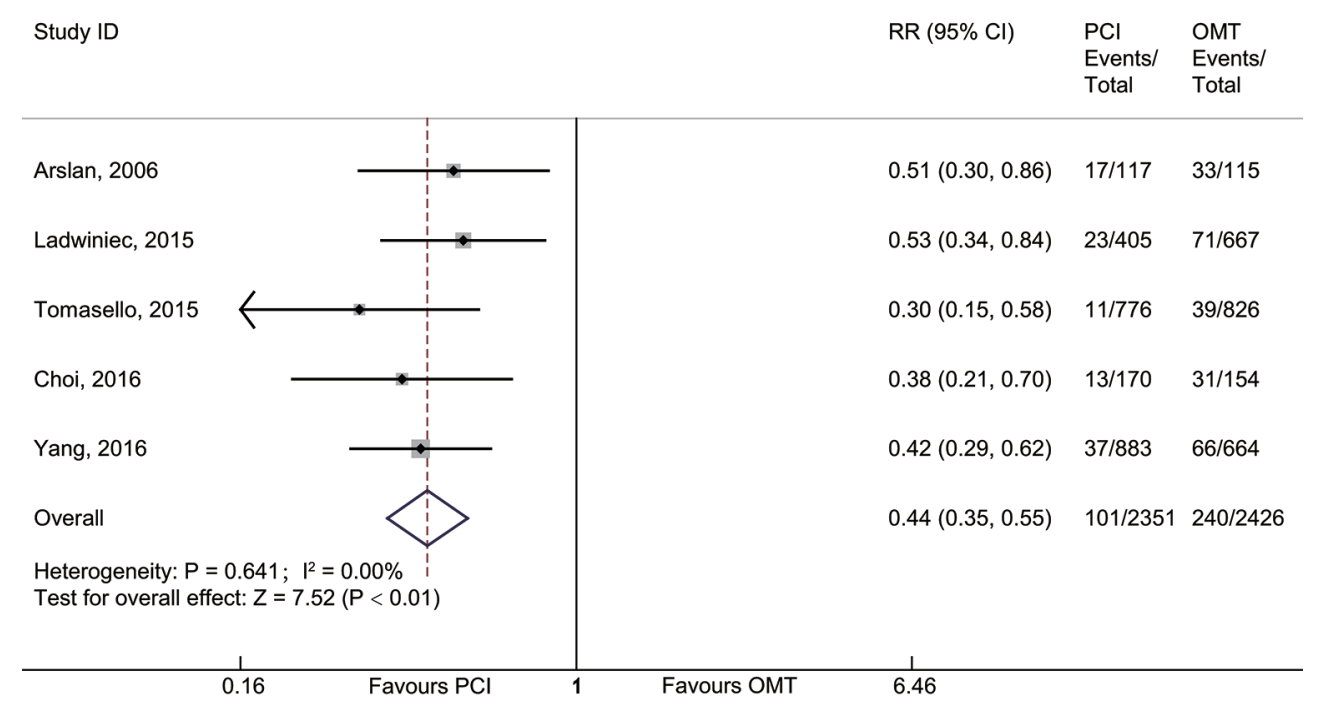

Figure 2 Forest plot with individual and summary estimates of the RR and 95\% CI of cardiac death in the main analysis. RR, risk ratio; CI, confidence interval; PCI, percutaneous coronary intervention; OMT, optimal medical therapy.

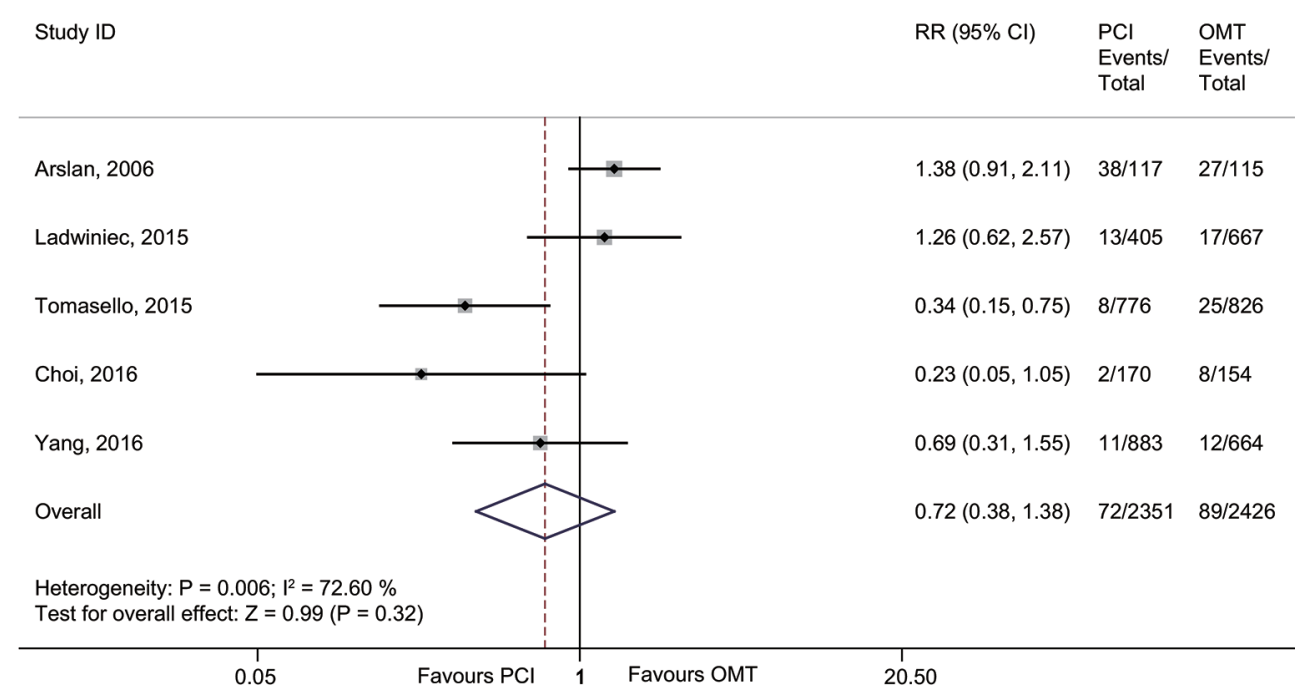

Figure 3 Forest plot with individual and summary estimates of the RR and 95\% CI of MI in the main analysis. RR, risk ratio; CI, confidence interval; PCI, percutaneous coronary intervention; OMT, optimal medical therapy; MI, myocardial infarction.

in MI (RR 0.72, 95\% CI: 0.38-1.38) between PCI and OMT groups (Figure 3). And PCI did not prevent stroke (RR 0.40, 95\% CI: 0.11-1.47) (Figure 4). In addition, the rate of MACE in patients undergoing PCI was lower (RR 0.64, 95\% CI: 0.43-0.97) in comparison with OMT (Figure 5).

A total of 3,082 patients were enrolled in the propensitymatched subgroup. The adopted therapeutic strategies were as follows: PCI in 1,542 patients and OMT in 1,542 patients. This analysis indicated that PCI reduced risk of cardiac death (RR $0.57,95 \%$ CI: $0.26-0.89$ ) by $43 \%$ when compared with OMT. Meanwhile, PCI prevented the occurrence of MI (RR 0.54, 95\% CI: 0.32-0.77). The rate of MACE in PCI group was lower (RR 0.76, 95\% CI: $0.33-1.18$ ) than that in OMT group but did not quite reach conventional statistical significance (Table S2).

After exclusion of COREA-AMI study (15), a total of 4,453 participants were included in the IRA subgroup, of 


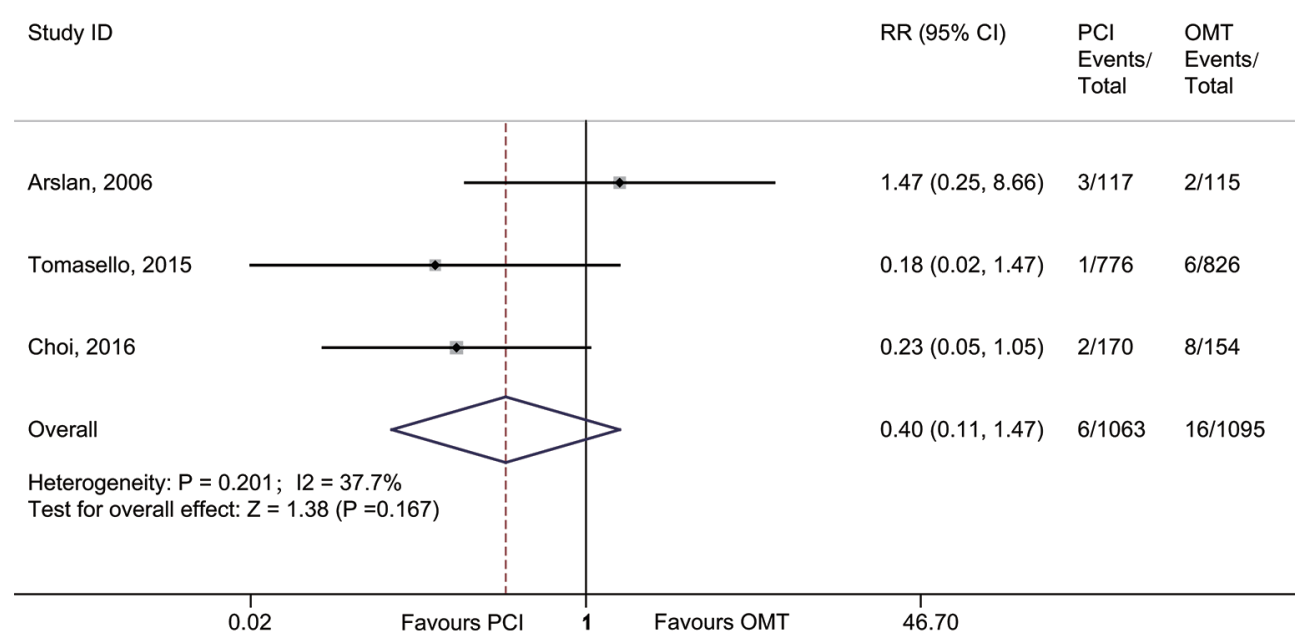

Figure 4 Forest plot with individual and summary estimates of the RR and 95\% CI of stroke in the main analysis. PCI, percutaneous coronary intervention; OMT, optimal medical therapy; RR, risk ratio; CI, confidence interval.

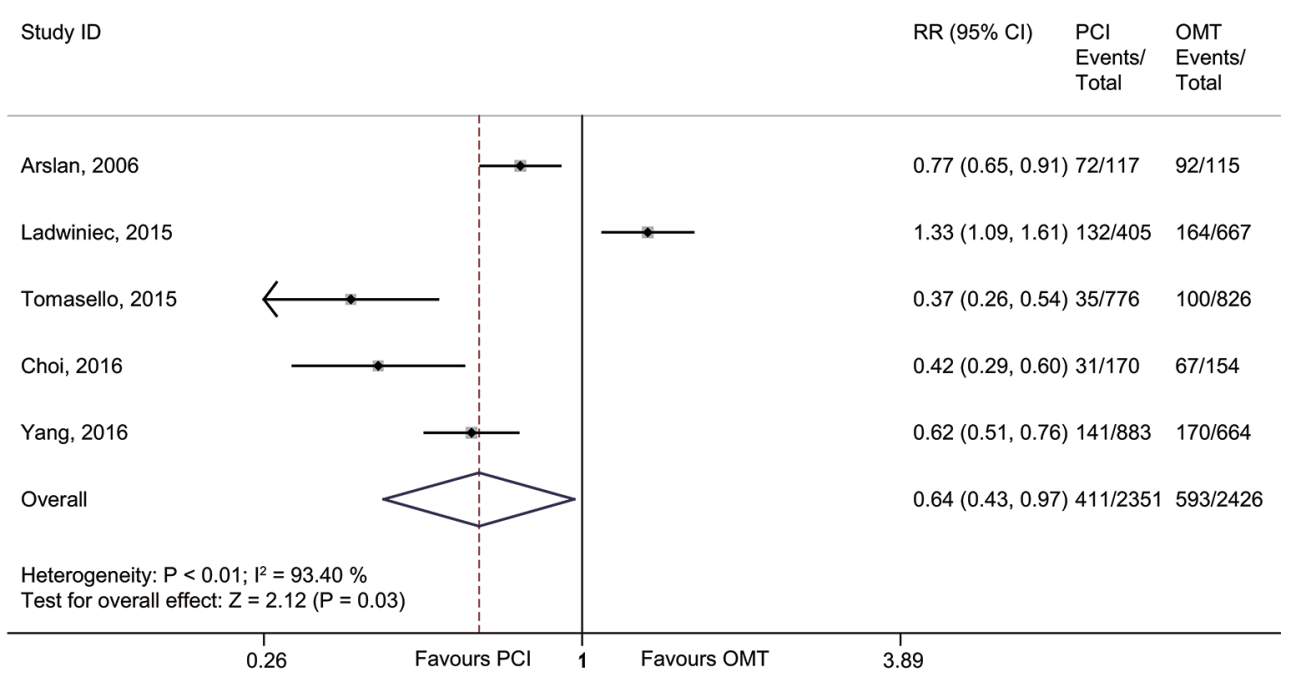

Figure 5 Forest plot with individual and summary estimates of the RR and 95\% CI of MACE in the main analysis. PCI, percutaneous coronary intervention; OMT, optimal medical therapy; RR, risk ratio; CI, confidence interval; MACE, major adverse cardiac events.

which there were 2,181 patients in PCI group and 2,272 patients in OMT group. This analysis demonstrated that PCI reduced risks of all-cause death (RR $0.41,95 \%$ CI: $0.34-0.49$ ) and cardiac death (RR 0.44, 95\% CI: 0.35-0.56) (Figures $S 2, S 3)$. But no difference was observed in MI between PCI and OMT groups (RR 0.84, 95\% CI: $0.45-$ 1.59) (Figure S4). In this subgroup analysis, when compared with OMT, PCI seemed to reduce occurrence of MACE (RR $0.71,95 \%$ CI: 0.46-1.10) with a tendency towards statistical significance (Figure S5).
Effect sizes reported in studies were distributed symmetrically (Figure S6) and there was no significant bias from small studies (Begg's test $\mathrm{P}=0.81$; Egger's test $\mathrm{P}=0.84$ ).

\section{Sensitivity analyses and quality assessment}

Jackknife sensitivity analyses (Table S3) showed that two studies significantly affected the pooled RRs of stroke and MACE. Exclusion of studies of Tomasello et al. (14) and Choi et al. (15) significantly increased risk of stroke. 
And PCI significantly reduced risk of MACE only after excluding study of Ladwiniec et al. (13).

The total scores of NOS of all studies were more than 8 points, which suggested that the overall risk of reporting bias was low and studies enrolled in this meta-analysis were reliable and good in quality (Table S4).

\section{Discussion}

In this meta-analysis, we observed that compared with OMT, PCI reduced risks of all-cause death, cardiac death and MACE. But there were no significant differences in MI and stroke between PCI and OMT groups. In addition, results of propensity-matched subgroup were in accordance with those of the main analysis, indicating that PCI reduced cardiac death, MI and MACE. In IRA subgroup, the rates of all-cause death, cardiac death and MACE in OMT group were higher than those in PCI group.

As shown in Table S1, although all the included studies were cohort studies, there were no significant differences in most of baseline variables between two groups. This indicated the baseline data of overall studies between PCI and OMT groups were well balanced and the comparability of two groups was good, which means lower bias. The average ages of patients included in these 5 studies were greater than 60 years old and over two thirds of patients were males, suggesting that CTO lesions were more common in elderly male patients. The proportion of patients with hypertension was high, which indicated that hypertension might play a role in the development of CTO. And a number of patients with hyperlipidemia were observed, confirming the role of lipid in the development of atherosclerosis. In addition, the proportion of patients with CTO in right coronary artery in OMT groups was higher than that in PCI groups while the proportion of patients whose left coronary artery and its branches were involved in PCI groups was higher. Based on the results of this meta-analysis, we might speculate that the benefit of PCI therapy in patients with CTO in left coronary artery and its branches was more obvious.

According to a meta-analysis (11) by Pursnani et al., as an initial treatment strategy in patients with stable coronary artery disease, PCI was associated with no improvement in all-cause death, cardiac death and $\mathrm{MI}$ as compared with OMT. However, CTO patients were not probably included in some studies identified in this metaanalysis due to difficulties in CTO PCI techniques. In contrast, a meta-analysis (20) that compared successful
PCI with failed PCI in patients with CTO reported successful PCI was associated with lower long-term allcause death and MI, confirming the role of successful PCI in the treatment of CTO patients. Nevertheless, some cardiologists doubted that complications related to failed PCI made the results of this analysis in favor of successful PCI and considered the results were "false" positive. When the initial management strategy was PCI or OMT, Arslan et al. (17) found PCI lowered the rates of all-cause death, cardiac death and MACE. Meanwhile, IRCTO study (14) demonstrated PCI improved survival and prevented MACE after 1-year follow-up. The benefit of PCI in mortality improvement was also observed in patients with CTO in non-IRA (15). Our results of the main analysis confirmed the data supporting the importance of CTO PCI. Compared with OMT, PCI reduced risks of all-cause death, cardiac death and MACE. The results of propensitymatched subgroup were consistent with those of the main analysis, in which effect of propensity-matching was extremely close to that of randomization. This strongly supported the results of main analysis, suggesting that PCI was associated with improved long-term survival in CTO patients.

Gao et al. (20) reported successful PCI with drug-eluting stents reduced risk of MI when compared with failed PCI. However, EXPLORE trial (8) found that the rate of MI in PCI group was similar to that in OMT group $(\mathrm{P}=0.49)$. In this meta-analysis, we didn't observe significant differences in MI and stroke between PCI and OMT groups, either. This might be related to high proportion of CTO patients with hyperlipidemia or hypertension in this analysis, which implied heavy burden of atherosclerosis in these patients. Additionally, restenosis risk of patients undergoing PCI procedures was relatively high in two studies $(13,17)$ because they were treated with bare-mental stents, leading to no significant reduction in MI in PCI group.

In IRA subgroup, PCI was associated with a significant decrease in mortality. And PCI reduced risk of MACE with a trend towards statistical significance. The results further supported the role of PCI in the CTO treatment.

Positive results were found in this analysis when the outcomes of PCI and OMT were compared. Recently, ORBITA trial (21) has been published and it showed PCI did not improve exercise time in patients with stable angina when compared with placebo procedure. But the duration of follow-up was only 6 weeks which was short. And exercise time was subjective as the primary endpoint. We thought it's better for investigators of this 
trial (21) to choose "hard endpoints", such as mortality or MACE. Meanwhile, two randomized controlled trials, EXPLORE (8) and DECISION-CTO (22) trials, failed to find PCI was superior to OMT in CTO patients. No differences in LVEF and left ventricular end diastolic volume (LVEDV) were observed between two groups after 4-month follow-up in the EXPLORE trial (8), demonstrating PCI didn't benefit CTO patients. But some limitations were presented in the EXPLORE trial. First of all, revascularization levels of PCI in CTO patients were unknown. Second, the duration of follow-up was just 4 months, which was relatively short. Moreover, this study enrolled acute ST-elevation MI patients, who were only a small part of CTO patients. DECISION-CTO trial (22) also failed to demonstrate difference in primary composite endpoint between PCI and OMT groups. The estimated sample size was 1,284 ; however, the trial was prematurely stopped due to difficulties to enroll patients. The final number of included patients was 834 , resulting in decreasing the test power. In addition, the rate of all-cause death in PCI group was lower than that in OMT group with a tendency towards statistical significance $(3.0 \% v s$. $4.4 \%$ at 3 -year follow-up, $4.5 \%$ vs. $7.9 \%$ at 5 -year followup). Hence, we needed to doubt whether results of these two trials were "true" negative.

OPEN-CTO Registry (23) showed that Seattle Angina Questionnaire quality of life scores in patients undergoing hybrid approach were improved and both of Rose Dyspnea Scale scores and physician health questionnaire scores in these patients were decreased. In the meantime, APPROACH registry (24) indicated life quality of patients who accepted PCI or coronary artery bypass grafting was better than that of patients accepting OMT. These two studies confirmed that PCI not only lowered the mortality of CTO patients but also relieved symptoms and improved life quality.

\section{Study limitations}

Firstly, this meta-analysis lacked specified individual data to conduct subgroup analyses. But the heterogeneity of most analyses was small and there was no need to explore the source of heterogeneity. Secondly, we could not make the subgroup analyses comparing the effects of PCI versus OMT in patients whose CTO located at different coronary arteries due to lacking corresponding outcome data. Thirdly, the studies included in our meta-analysis did not report economic data, resulting in failure to make the cost-effectiveness analysis. But cost-efficacy is increasingly important in the choice of therapy strategies. Thus, more researches concerning cost-effectiveness are expected.

\section{Conclusions}

PCI, as compared with OMT, was associated with significant improvement in mortality and MACE. Therefore, PCI was recommended for CTO patients.

\section{Acknowledgements}

Thank Hui Xie for his assistance on graphing.

\section{Footnote}

Conflicts of Interest: The authors have no conflicts of interest to declare.

\section{References}

1. Windecker S, Kolh P, Alfonso F, et al. 2014 ESC/EACTS Guidelines on myocardial revascularization: The Task Force on Myocardial Revascularization of the European Society of Cardiology (ESC) and the European Association for Cardio-Thoracic Surgery (EACTS)Developed with the special contribution of the European Association of Percutaneous Cardiovascular Interventions (EAPCI). Eur Heart J 2014;35:2541-619.

2. Levine GN, Bates ER, Blankenship JC, et al. 2011 ACCF/AHA/SCAI Guideline for Percutaneous Coronary Intervention. A report of the American College of Cardiology Foundation/American Heart Association Task Force on Practice Guidelines and the Society for Cardiovascular Angiography and Interventions. J Am Coll Cardiol 2011;58:e44-122.

3. Fefer P, Knudtson ML, Cheema AN, et al. Current perspectives on coronary chronic total occlusions: the Canadian Multicenter Chronic Total Occlusions Registry. J Am Coll Cardiol 2012;59:991-7.

4. Christofferson RD, Lehmann KG, Martin GV, et al. Effect of chronic total coronary occlusion on treatment strategy. Am J Cardiol 2005;95:1088-91.

5. Werner GS, Gitt AK, Zeymer U, et al. Chronic total coronary occlusions in patients with stable angina pectoris: impact on therapy and outcome in present day clinical practice. Clin Res Cardiol 2009;98:435-41.

6. George S, Cockburn J, Clayton TC, et al. Long-term 
follow-up of elective chronic total coronary occlusion angioplasty: analysis from the U.K. Central Cardiac Audit Database. J Am Coll Cardiol 2014;64:235-43.

7. Michael TT, Karmpaliotis D, Brilakis ES, et al. Procedural outcomes of revascularization of chronic total occlusion of native coronary arteries (from a multicenter United States registry). Am J Cardiol 2013;112:488-92.

8. Henriques JP, Hoebers LP, Ramunddal T, et al. Percutaneous Intervention for Concurrent Chronic Total Occlusions in Patients With STEMI: The EXPLORE Trial. J Am Coll Cardiol 2016;68:1622-32.

9. Galassi AR, Boukhris M, Tomasello SD, et al. Long-term clinical and angiographic outcomes of the mini-STAR technique as a bailout strategy for percutaneous coronary intervention of chronic total occlusion. Can J Cardiol 2014;30:1400-6.

10. Olivari Z, Rubartelli P, Piscione F, et al. Immediate results and one-year clinical outcome after percutaneous coronary interventions in chronic total occlusions: data from a multicenter, prospective, observational study (TOASTGISE). J Am Coll Cardiol 2003;41:1672-8.

11. Pursnani S, Korley F, Gopaul R, et al. Percutaneous coronary intervention versus optimal medical therapy in stable coronary artery disease: a systematic review and meta-analysis of randomized clinical trials. Circ Cardiovasc Interv 2012;5:476-90.

12. Paul S, Lee PC, Mao J, et al. Long term survival with stereotactic ablative radiotherapy (SABR) versus thoracoscopic sublobar lung resection in elderly people: national population based study with propensity matched comparative analysis. BMJ 2016;354:i3570.

13. Ladwiniec A, Allgar V, Thackray S, et al. Medical therapy, percutaneous coronary intervention and prognosis in patients with chronic total occlusions. Heart 2015;101:1907-14.

14. Tomasello SD, Boukhris M, Giubilato S, et al. Management strategies in patients affected by chronic total occlusions: results from the Italian Registry of Chronic Total Occlusions. Eur Heart J 2015;36:3189-98.

15. Choi IJ, Koh YS, Lim S, et al. Impact of Percutaneous

Cite this article as: Ma Y, Li D, Li J, Li Y, Bai F, Qin F, Zhou S, Liu Q. Percutaneous coronary intervention versus optimal medical therapy for patients with chronic total occlusion: a meta-analysis and systematic review. J Thorac Dis 2018;10(5):2960-2967. doi: 10.21037/jtd.2018.04.140
Coronary Intervention for Chronic Total Occlusion in Non-Infarct-Related Arteries in Patients With Acute Myocardial Infarction (from the COREA-AMI Registry). Am J Cardiol 2016;117:1039-46.

16. Yang JH, Kim BS, Jang WJ, et al. Optimal Medical Therapy vs. Percutaneous Coronary Intervention for Patients With Coronary Chronic Total Occlusion - A Propensity-Matched Analysis. Circ J 2016;80:211-7.

17. Arslan U, Balcioglu AS, Timurkaynak T, et al. The clinical outcomes of percutaneous coronary intervention in chronic total coronary occlusion. Int Heart J 2006;47:811-9.

18. Renda G, Ricci F, Giugliano RP, et al. Non-Vitamin K Antagonist Oral Anticoagulants in Patients With Atrial Fibrillation and Valvular Heart Disease. J Am Coll Cardiol 2017;69:1363-71.

19. Stang A. Critical evaluation of the Newcastle-Ottawa scale for the assessment of the quality of nonrandomized studies in meta-analyses. Eur J Epidemiol 2010;25:603-5.

20. Gao L, Wang Y, Liu Y, et al. Long-term clinical outcomes of successful revascularization with drug-eluting stents for chronic total occlusions: A systematic review and metaanalysis. Catheter Cardiovasc Interv 2017;89:574-581.

21. Al-Lamee R, Thompson D, Dehbi HM, et al. Percutaneous coronary intervention in stable angina (ORBITA): a double-blind, randomised controlled trial. Lancet 2018;391:31-40.

22. Park SJ. Optimal Medical Therapy With or Without Stenting for Coronary Chronic Total Occlusion: DECISION-CTO. Paper presented at: American College Cardiology Congress, Washington, DC, 2017.

23. Sapontis J, Salisbury AC, Yeh RW, et al. Early procedural and health status outcomes after chronic total occlusion angioplasty: a report from the OPEN-CTO Registry. JACC Cardiovasc Interv 2017;10:1523-34.

24. Wijeysundera HC, Norris C, Fefer P, et al. Relationship between initial treatment strategy and quality of life in patients with coronary chronic total occlusions. EuroIntervention 2014;9:1165-72. 


\section{Supplement methods}

\section{Search strategy}

\section{PubMed database}

\#1 "chronic total occlusion" OR "chronic total coronary occlusion" OR "coronary chronic total occlusion" OR "chronic coronary total occlusion"

\#2 "Angioplasty, balloon, coronary" [MeSH] OR "Percutaneous transluminal coronary angioplasty" OR "Angioplasty, Balloon, Coronary" [MeSH Terms] OR "Cardiac catheterization" OR "Heart catheterization" OR "Drug-eluting stent" OR "Drug-eluting stents" OR "Drug eluting stent" OR "Bare metal stent" OR "Percutaneous coronary intervention" OR "PCI" OR "PTCA" OR "Angioplasty"

\#3 "Conservative therapy" OR "Medical therapy" OR "Antihypertensive agents" [MeSH Terms] OR "Antihypertensive agent*" OR "Calcium channel blockers" [MeSH Terms] OR "Calcium channel blocker" OR "Calcium channel blockers" OR "Aspirin" OR "Nitroglycerin" OR "Nitric oxide donors"[MeSH Terms] OR "Nitric oxide donor" OR "Nitric oxide donors" OR "Anticholesteremic agents"[MeSH Terms] OR "Anticholesteremic" OR "Lipid regulating agents" [MeSH Terms] OR "Lipid regulating agents" OR "exercise therapy" [MeSH Terms] OR "Statin" OR "Statins" \#4 \#2 OR \#3

\#5 \#1 AND \#4

\section{Embase database}

\#1 'chronic total occlusion' OR 'chronic total coronary occlusion' OR 'coronary chronic total occlusion' OR 'chronic coronary total occlusion'

\#2 'transluminal coronary angioplasty' OR 'heart catheterization' OR 'drug eluting stent' OR 'bare metal stent' OR 'coronary stent' OR 'percutaneous coronary intervention' OR 'percutaneous transluminal coronary angioplasty' OR 'cardiac catheterization' OR 'ptca' OR 'pci'

\#3 'antihypertensive agent' OR 'calcium channel blocking agent' OR aspirin OR 'nitric oxide donor' OR 'antilipemic agent' OR statins OR statin OR 'conservative treatment' OR 'medical therapy'

\#4 \#2 OR \#3

\#5\#1 AND \#4

\section{Cochrane library}

\#1 "chronic total occlusion" OR "chronic total coronary occlusion" OR "coronary chronic total occlusion" OR "chronic coronary total occlusion"

\#2 MeSH descriptor: [Percutaneous Coronary Intervention] explode all trees

\#3 MeSH descriptor: [Drug-Eluting Stents] explode all trees

\#4 "Cardiac catheterization" or "Heart catheterization" or "Drug eluting stent" or "Bare metal stent" or "PCI" or "PTCA" or "Angioplasty"

\#5 \#2 OR \#3 OR \#4

\#6 MeSH descriptor: [Antihypertensive Agents] explode all trees

\#7 MeSH descriptor: [Calcium Channel Blockers] explode all trees

\#8 MeSH descriptor: [Nitric Oxide Donors] explode all trees

\#9 MeSH descriptor: [Lipid Regulating Agents] explode all trees

\#10 MeSH descriptor: [Anticholesteremic Agents] explode all trees

\#11 MeSH descriptor: [Exercise Therapy] explode all trees

\#12 "Conservative therapy" OR "Medical therapy" OR "Antihypertensive agent*" OR "Calcium channel blockers" OR "Calcium channel blocker" OR "Calcium channel blockers" OR "Aspirin" OR "Nitroglycerin" OR "Nitric oxide donor" OR "Nitric oxide donors" OR "Anticholesteremic" OR "Lipid regulating agents" OR "Statin" or "Statins"

\#13 \#6 OR \#7 OR \#8 OR \#9 OR \#10 OR \#11 OR \#12 \#14 \#5 OR \#13

\#15 \#1 AND \#14 


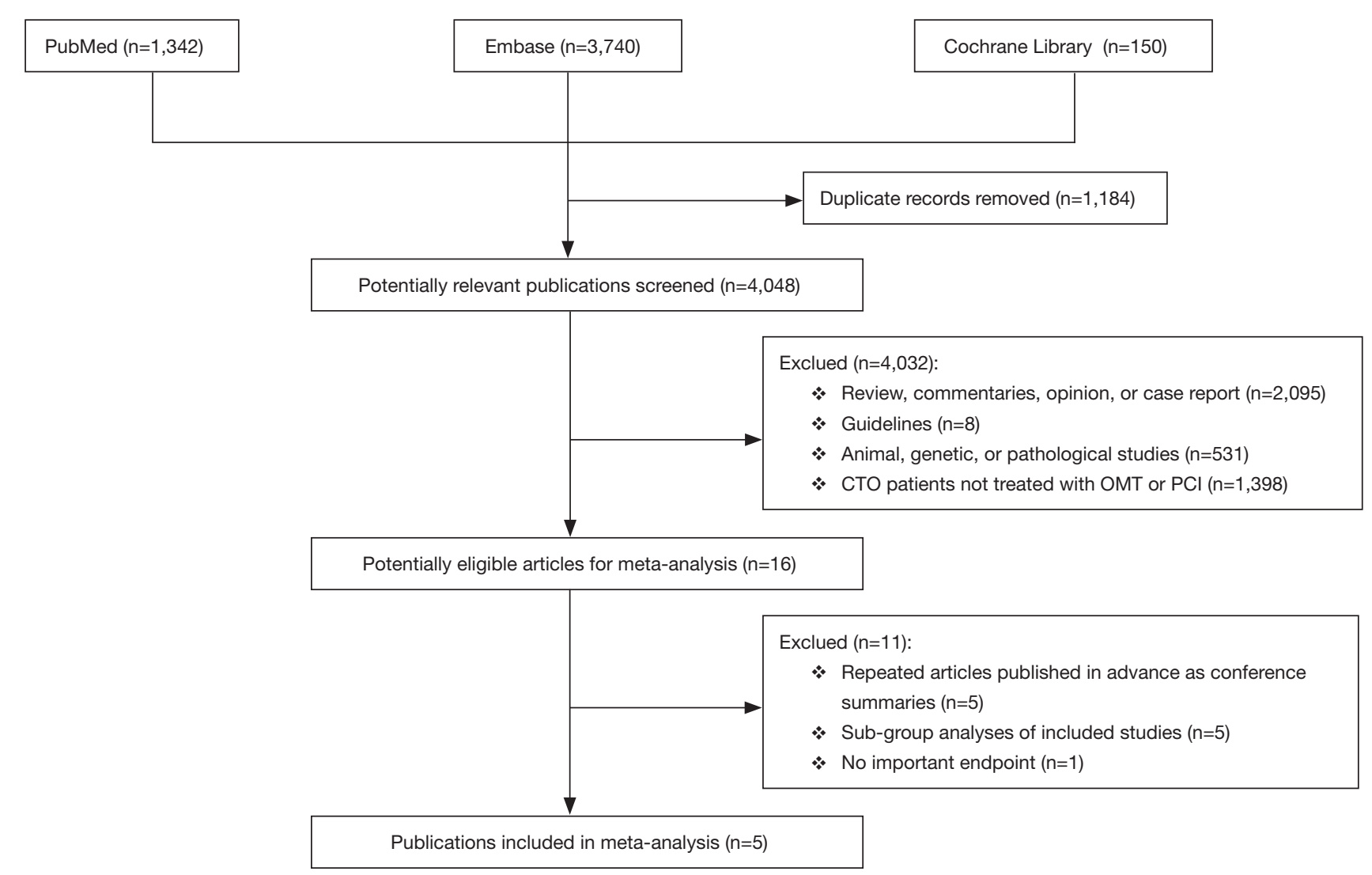

Figure S1 Flow chart showing the process of study selection and numbers of studies included. CTO, chronic total occlusion; PCI, percutaneous coronary intervention; OMT, optimal medical therapy.

Table S1 Baseline characteristics of the populations of the included studies

\begin{tabular}{|c|c|c|c|c|c|c|c|c|c|c|}
\hline \multirow{2}{*}{ Variables } & \multicolumn{2}{|c|}{ Arslan, 2006} & \multicolumn{2}{|c|}{ Ladwiniec, 2015} & \multicolumn{2}{|c|}{ Tomasello, 2015} & \multicolumn{2}{|c|}{ Choi, 2016} & \multicolumn{2}{|c|}{ Yang, 2016} \\
\hline & $\mathrm{PCl}(\mathrm{n}=117)$ & OMT $(n=115)$ & $\mathrm{PCl}(\mathrm{n}=405)$ & OMT $(n=651)$ & $\mathrm{PCl}(\mathrm{n}=776)$ & OMT $(n=826)$ & $\mathrm{PCl}(\mathrm{n}=170)$ & OMT $(n=154)$ & $\mathrm{PCl}(\mathrm{n}=883)$ & OMT $(n=664)$ \\
\hline Age (years) & $61.1 \pm 10.4$ & $60.3 \pm 10.6$ & $63.2 \pm 10.1$ & 65. $8 \pm 10.7^{*}$ & $67.0 \pm 10.6$ & $70.1 \pm 12.5^{\star}$ & $62.7 \pm 12.9$ & $67.5 \pm 11.2^{*}$ & $61.5 \pm 10.8$ & $65.9 \pm 11.3^{\star}$ \\
\hline Male (\%) & $88(75.2)$ & $86(74.8)$ & 301 (73.1) & $506(77.7)$ & $658(84.8)$ & $690(83.5)$ & 118 (69.4) & 102 (66.2) & $713(80.7)$ & $509(76.7)$ \\
\hline Diabetes (\%) & $30(25.6)$ & 27 (23.5) & 78 (19.3) & $137(21.0)$ & 232 (29.9) & $245(29.7)$ & $56(32.9)$ & $53(34.4)$ & $384(43.5)$ & 318 (47.9) \\
\hline Hypertension (\%) & $47(40.2)$ & $49(42.6)$ & $225(55.6)$ & 349 (53.6) & 604 (77.8) & $645(78.1)$ & $93(54.7)$ & $88(57.1)$ & 547 (61.9) & $439(66.1)$ \\
\hline Dyslipidemia (\%) & $31(26.5)$ & 27 (23.5) & $199(49.1)$ & $293(45.0)$ & $476(61.3)$ & $533(64.5)$ & 107 (62.9) & $99(64.3)$ & $289(32.7)$ & $158(23.8)^{\star}$ \\
\hline Stroke (\%) & $(-)$ & $(-)$ & $22(5.4)$ & $61(9.4)^{\star}$ & $(-)$ & $(-)$ & $8(4.7)$ & $7(4.6)$ & $66(7.5)$ & $67(10.1)$ \\
\hline Prior MI (\%) & $47(40.2)$ & $52(45.2)$ & 202 (49.9) & $394(60.5)^{\star}$ & $318(41)$ & $372(45)$ & $9(5.3)$ & $6(3.9)$ & $180(20.4)$ & $211(31.8)^{\star}$ \\
\hline Smoking (\%) & $81(69.2)$ & $83(72.2)$ & $273(67.4)$ & 466 (71.5) & $324(41.8)$ & 384 (46.5) & $59(34.7)$ & $54(35.1)$ & 285 (32.3) & $182(27.4)$ \\
\hline \multicolumn{11}{|l|}{ СТO vessels } \\
\hline LAD (\%) & $35(29.9)$ & 34 (29.6) & $53(13.1)^{\#}$ & $90(13.8)^{\#}$ & $(-)$ & $(-)$ & 57 (33.5) & $39(25.6)$ & 373 (42.2) & $168(25.3)^{\star}$ \\
\hline LCX (\%) & 49 (41.9) & 44 (38.3) & $42(10.4)^{\S}$ & $115(17.7)^{\star \S}$ & $(-)$ & $(-)$ & $56(32.9)$ & $63(40.9)$ & 261 (29.6) & 228 (34.3) \\
\hline RCA (\%) & 33 (28.2) & 37 (32.2) & & & $(-)$ & $(-)$ & 57 (33.5) & 52 (33.8) & $383(43.4)$ & $370(55.7)^{\star}$ \\
\hline
\end{tabular}

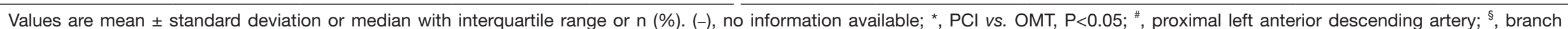

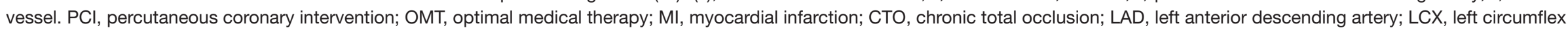
artery; RCA, right coronary artery. 
Table S2 Summary of data comparing PCI with OMT in patients included in the propensity-matched analysis subgroup

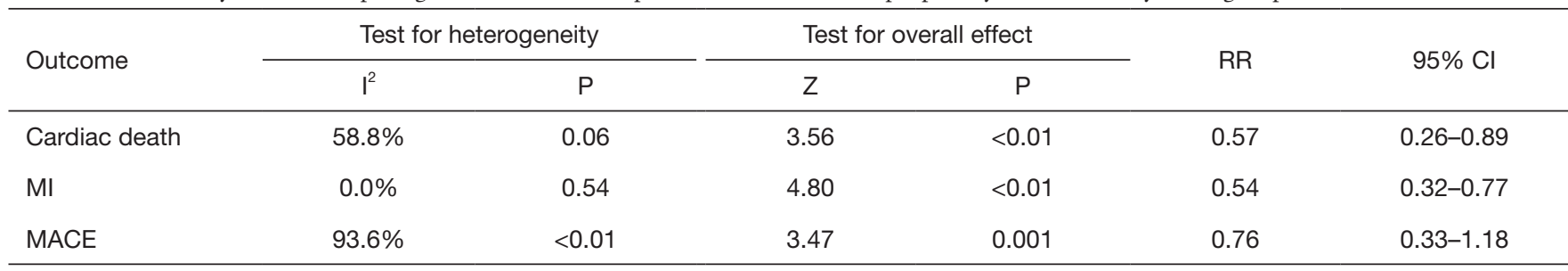

$\mathrm{PCl}$, percutaneous coronary intervention; OMT, optimal medical therapy; RR, risk ratio; Cl, confidence interval; MI, myocardial infarction; MACE, major adverse cardiac events.

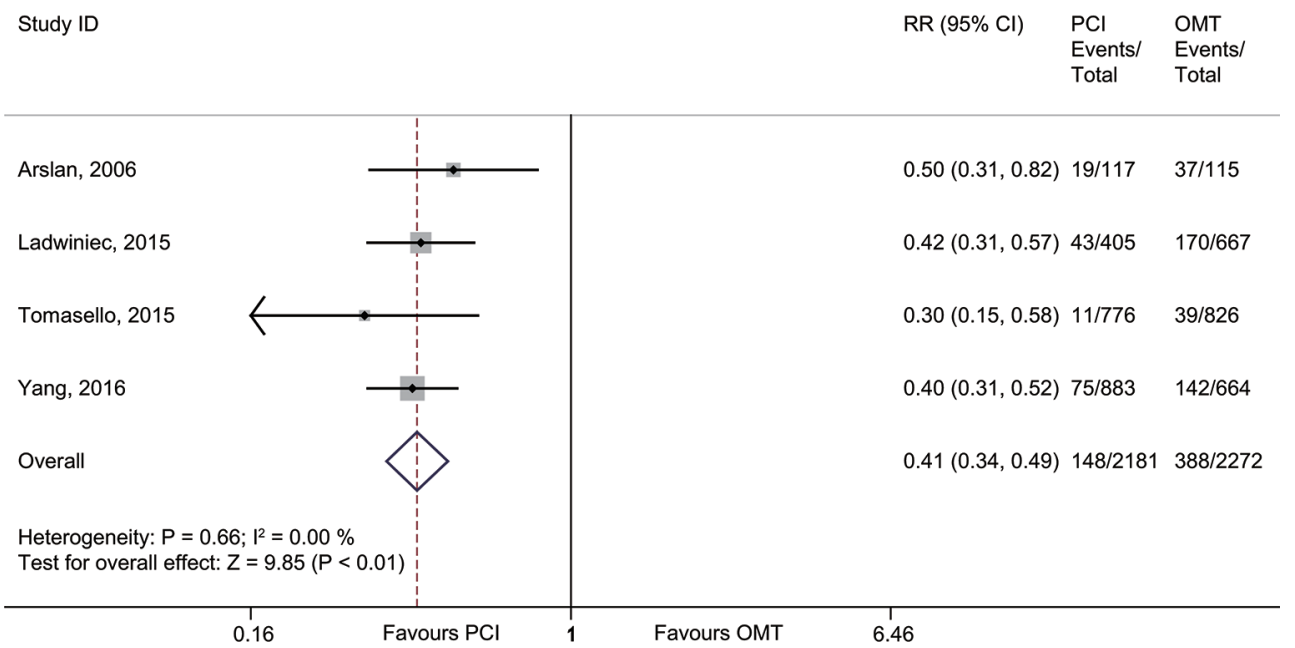

Figure S2 Forest plot with individual and summary estimates of the RR and 95\% CI of all-cause death in IRA subgroup. PCI, percutaneous coronary intervention; OMT, optimal medical therapy; RR, risk ratio; CI, confidence interval.

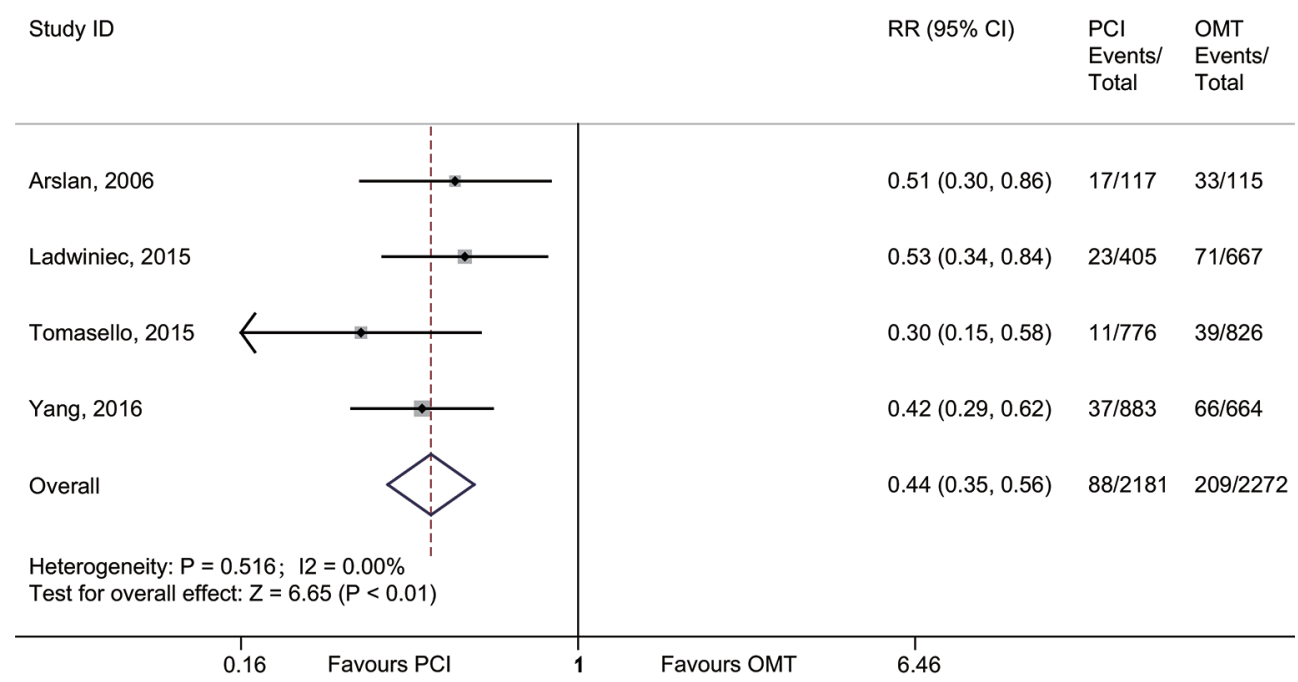

Figure S3 Forest plot with individual and summary estimates of the RR and 95\% CI of cardiac death in IRA subgroup. PCI, percutaneous coronary intervention; OMT, optimal medical therapy; IRA, infarct-related artery; RR, risk ratio; CI, confidence interval. 


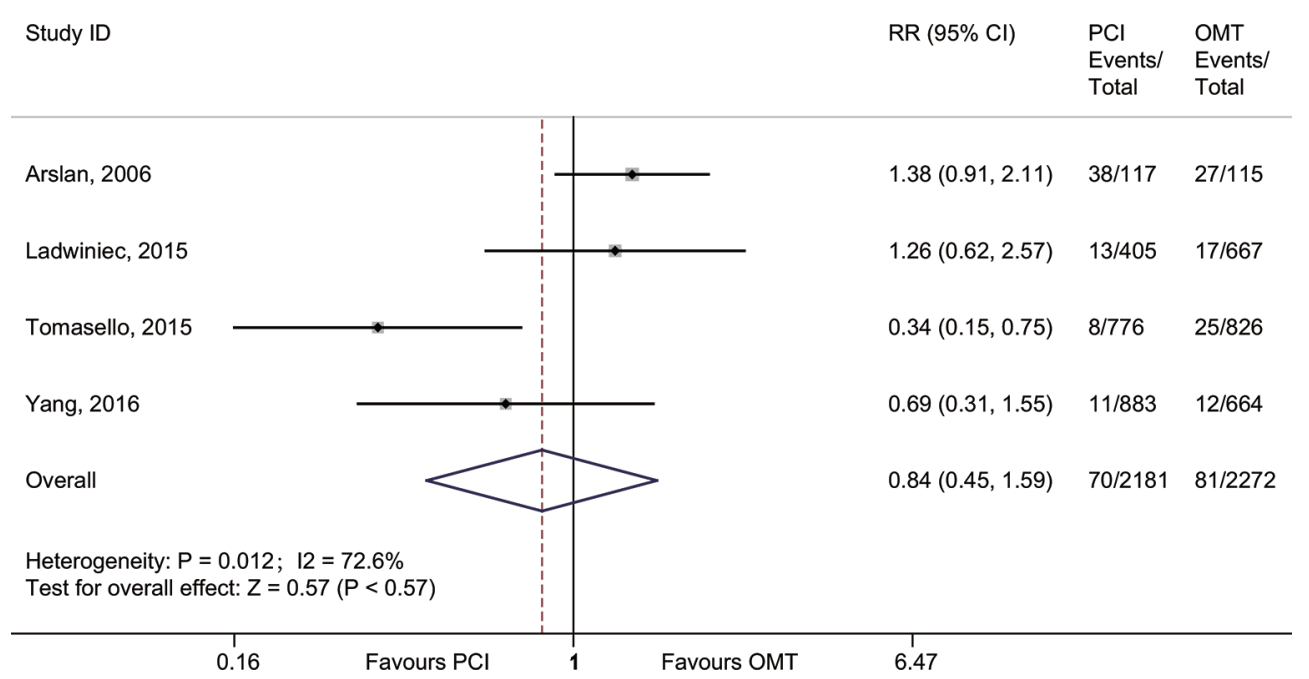

Figure S4 Forest plot with individual and summary estimates of the RR and 95\% CI of MI in IRA subgroup. PCI, percutaneous coronary intervention; OMT, optimal medical therapy; MI; myocardial infarction; IRA, infarct-related artery; RR, risk ratio; CI, confidence interval.

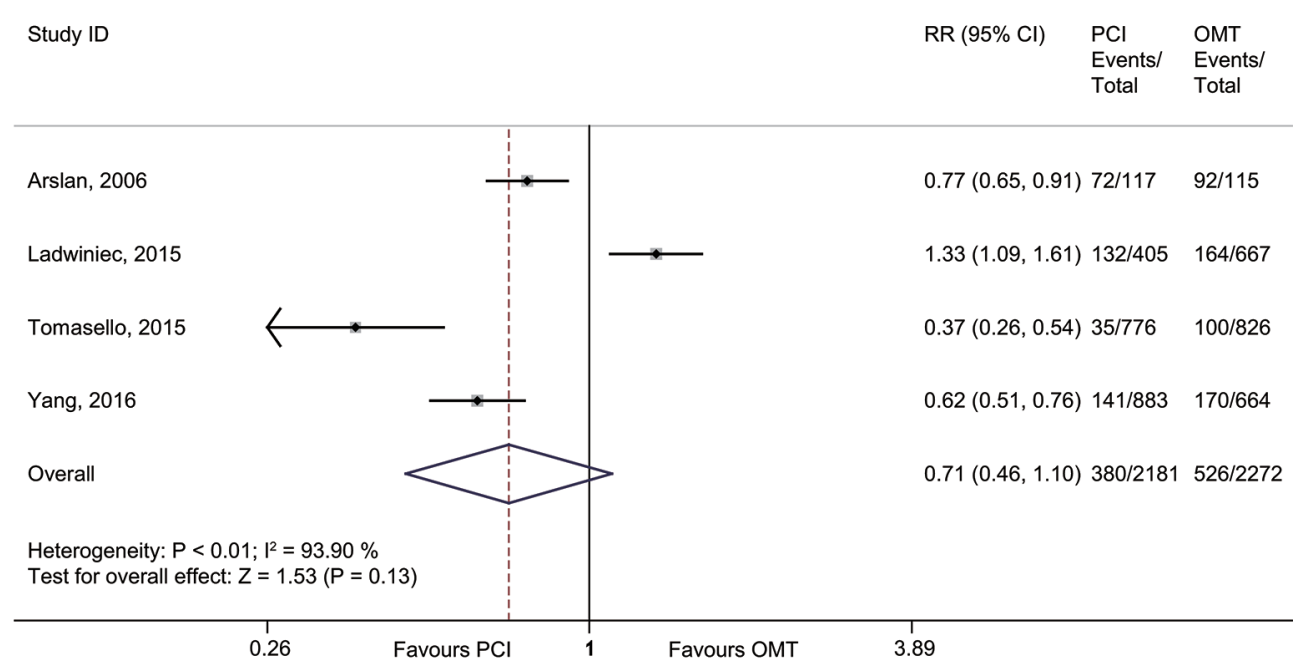

Figure S5 Forest plot with individual and summary estimates of the RR and 95\% CI of MACE in IRA subgroup. RR, risk ratio; CI, confidence interval; PCI, percutaneous coronary intervention; OMT, optimal medical therapy; MACE, major adverse cardiac events; IRA, infarct-related artery. 


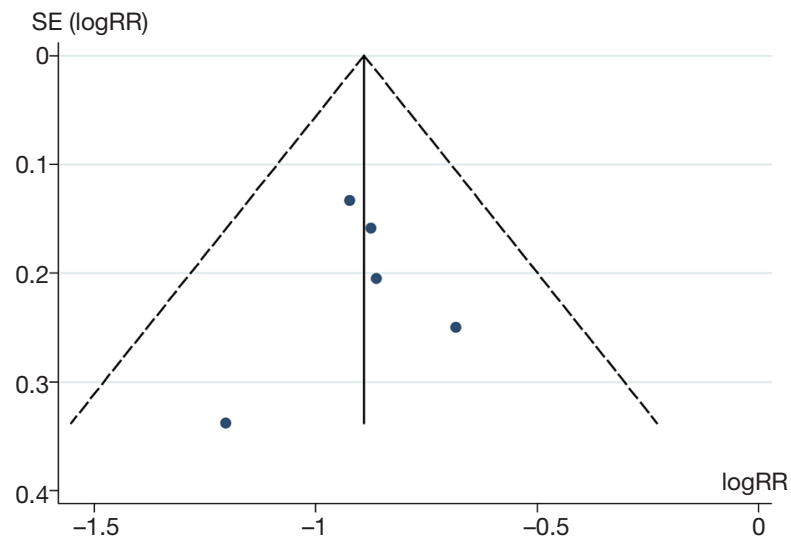

Figure S6 Assessment of publication bias by the funnel plot. SE, standard error; RR, risk ratio.

Table S3 Jackknife sensitivity analyses of studies

\begin{tabular}{|c|c|}
\hline Study & $\mathrm{RR}(95 \% \mathrm{Cl})$ \\
\hline \multicolumn{2}{|l|}{ All-cause death } \\
\hline Omitting Arslan, 2006 & $0.40(0.34,0.48)$ \\
\hline Omitting Ladwiniec, 2015 & $0.41(0.34,0.49)$ \\
\hline Omitting Tomasello, 2015 & $0.42(0.35,0.50)$ \\
\hline Omitting Choi, 2016 & $0.41(0.34,0.49)$ \\
\hline Omitting Yang, 2016 & $0.42(0.34,0.52)$ \\
\hline Subtotal & $0.41(0.35,0.48)$ \\
\hline \multicolumn{2}{|l|}{ Cardiac death } \\
\hline Omitting Arslan, 2006 & $0.39(0.30,0.51)$ \\
\hline Omitting Ladwiniec, 2015 & $0.37(0.28,0.48)$ \\
\hline Omitting Tomasello, 2015 & $0.42(0.32,0.55)$ \\
\hline Omitting Choi, 2016 & $0.41(0.31,0.53)$ \\
\hline Omitting Yang, 2016 & $0.40(0.29,0.54)$ \\
\hline Subtotal & $0.40(0.31,0.51)$ \\
\hline \multicolumn{2}{|l|}{ MI } \\
\hline Omitting Arslan, 2006 & $0.57(0.28,1.19)$ \\
\hline Omitting Ladwiniec, 2015 & $0.60(0.25,1.39)$ \\
\hline Omitting Tomasello, 2015 & $0.94(0.53,1.66)$ \\
\hline Omitting Choi, 2016 & $0.84(0.45,1.59)$ \\
\hline Omitting Yang, 2016 & $0.71(0.31,1.60)$ \\
\hline Subtotal & $0.72(0.38,1.38)$ \\
\hline
\end{tabular}

Table S3 (continued)

\begin{tabular}{ll}
\hline Study & $\mathrm{RR}(95 \% \mathrm{Cl})$ \\
\hline Stroke & \\
Omitting Arslan, 2006 & $0.20(0.06,0.71)$ \\
Omitting Tomasello,2015 & $0.46(0.16,1.35)$ \\
Omitting Choi, 2016 & $0.50(0.15,1.71)$ \\
Subtotal & $0.36(0.14,0.92)$ \\
MACE & \\
Omitting Arslan, 2006 & $0.61(0.34,1.09)$ \\
Omitting Ladwiniec, 2015 & $0.54(0.39,0.75)$ \\
Omitting Tomasello, 2015 & $0.73(0.48,1.11)$ \\
Omitting Choi, 2016 & $0.71(0.46,1.10)$ \\
Omitting Yang, 2016 & $0.64(0.38,1.10)$ \\
Subtotal & $0.64(0.43,0.97)$
\end{tabular}

Pooled estimates for all cause death, cardiac death, MI, stroke, and MACE were recalculated, using a random-effect model, by omitting one study at a time. Each line represents a re-analysis of the data with exclusion of one study at a time to assess the influence of this particular study on the overall result. Numbers in bold show the statistically significant differences. RR, risk ratio; Cl, confidence interval; $\mathrm{MI}$, myocardial infarction; MACE, major adverse cardiac events.

Table S3 (continued) 
Table S4 Quality assessment of the observational studies included in the meta-analysis by Newcastle-Ottawa scale

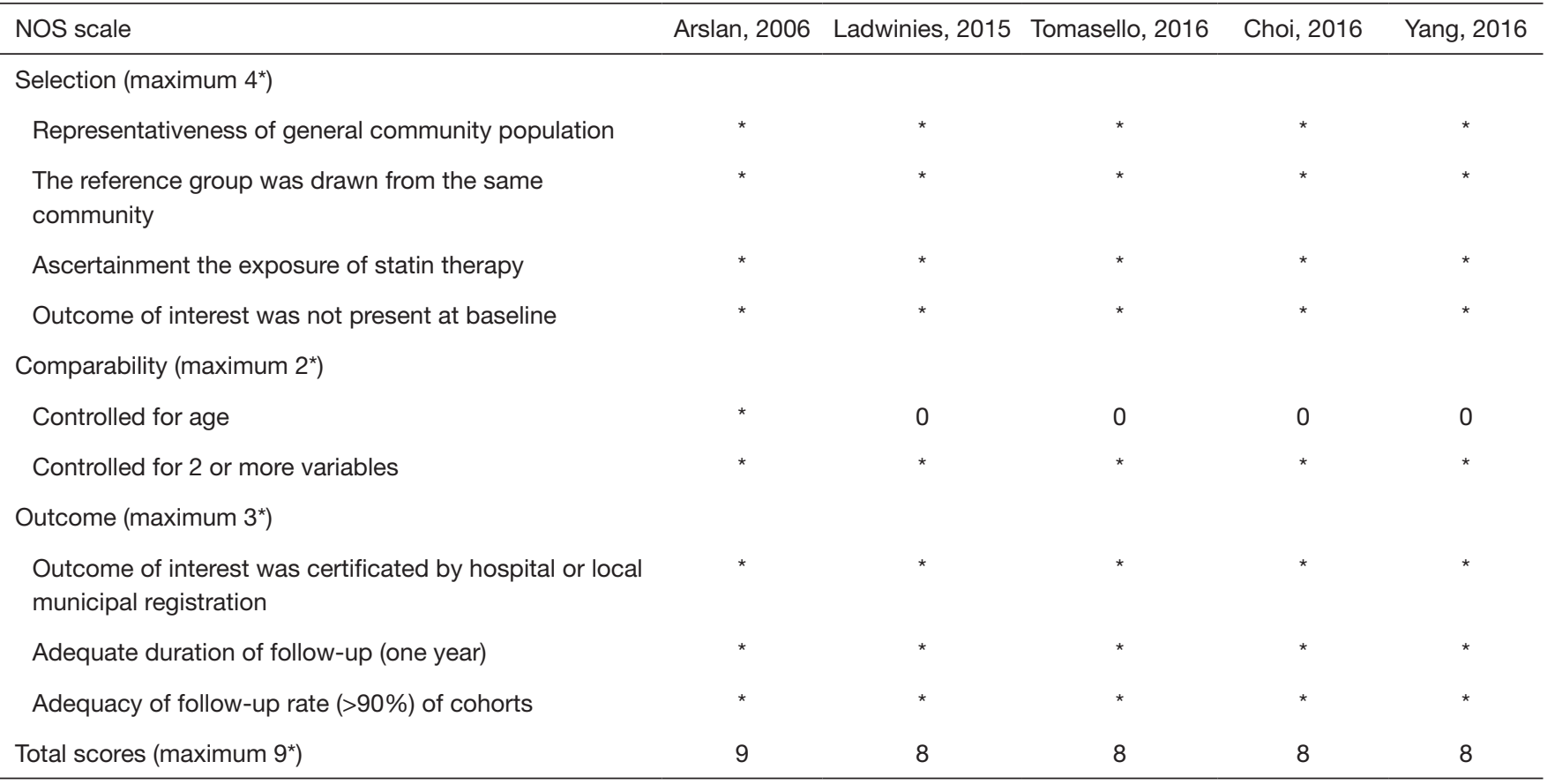

*, meant the study was corresponded to the Newcastle-Ottawa scale criteria; 0, meant the study wasn't corresponding to NewcastleOttawa scale criteria. NOS, Newcastle-Ottawa scale. 